\title{
Phylogenetic Placement of Dialister pneumosintes (formerly Bacteroides pneumosintes) within the Sporomusa Subbranch of the Clostridium Subphylum of the Gram-Positive Bacteria
}

\author{
ANNE WILLEMS* AND MATTHEW D. COLLINS \\ Department of Microbiology, Institute of Food Research, Reading Laboratory, Reading RG6 2EF, United Kingdom

\begin{abstract}
The nucleotide sequence of the 16S rRNA gene of the type strain of Dialister pneumosintes was determined. Phylogenetic analysis revealed that this species belongs to the Sporomusa branch of the Clostridium subphylum of the gram-positive bacteria and should therefore be excluded from the family Bacteroidaceae. Within this branch, which encompasses several other gram-negative taxa, such as Acidaminococcus, Pectinatus, Phascolarcobacterium, Quinella, Selenomonas, and Zymophilus, Dialister showed a specific, albeit distant, affinity with the genera Megasphaera and Veillonella.
\end{abstract}

The genus Dialister was recently revived to accommodate the gram-negative, obligately anaerobic, nonfermentative, rodshaped species Bacteroides pneumosintes (11). B. pneumosintes was originally described by Olitsky and Gates as Bacterium pneumosintes in 1921 (12) and was first isolated from nasopharyngeal secretions of patients with influenza during the epidemics of 1918 through 1921 (12). The species was later placed in the genus Dialister and subsequently transferred to the genus Bacteroides in 1970 (8). During the 1980s the genus Bacteroides has undergone major taxonomic revisions, with many of the species being transferred to new genera (viz., Anaerorhabdus, Fibrobacter, Megamonas, Mitsuokella, Porphyromonas, Prevotella, Rikenella, Ruminobacter, Sebaldella, and Tissierella) (see reference 13 for a review). Shah and Collins in 1989 (14) proposed that the genus Bacteroides be restricted to the saccharolytic, nonpigmented species of the Bacteroides fragilis group. B. pneumosintes differs markedly from this genus as defined by Shah and Collins (14), and its true taxonomic affiliations remained uncertain. On the basis of phenotypic criteria, Moore and Moore (11) have recently resurrected the genus Dialister, to accommodate $B$. pneumosintes. In this study, we have sequenced the 16S rRNA gene of the type strain of Dialister pneumosintes to determine its phylogenetic proximity to the genus Bacteroides and other members of the family Bacteroidaceae.

A freeze-dried culture of the type strain of $D$. pneumosintes (ATCC $33048^{\mathrm{T}}$ ) was obtained from the American Type Culture Collection, Rockville, Md. Because of the phenotypic similarities of Veillonella and Dialister species (e.g., small cell size and non-spore-forming, nonmotile anaerobes), the 16S rRNA genes of three Veillonella species from the human oral cavity were also sequenced. Veillonella atypica (DSM 20739 ${ }^{\mathrm{T}}$ ), Veillonella dispar (DSM $20735^{\mathrm{T}}$ ), and Veillonella parvula (DSM $2008^{\mathrm{T}}$ ) were obtained from the Deutsche Sammlung von Mikroorganismen und Zellkulturen, Braunschweig, Germany. Lyophilized cells were resuspended in $500 \mu$ l of TES buffer (0.05 M Tris- $\mathrm{HCl}, 0.005 \mathrm{M}$ EDTA, $0.05 \mathrm{M} \mathrm{NaCl}, \mathrm{pH} 8.0)$, and genomic DNA was extracted directly from this cell suspension by the method of Lawson et al. (10). The $16 \mathrm{~S}$ rRNA genes were amplified as described previously (9) and purified by using a Prep-A-Gene kit (Bio-Rad, Hercules, Calif.) according to the

${ }^{*}$ Corresponding author. Mailing address: Department of Microbiology, Institute of Food Research, Reading Laboratory, Earley Gate, Whiteknights Rd., Reading RG6 2EF, United Kingdom. manufacturer's instructions. The $16 \mathrm{~S}$ rDNA fragments were sequenced by using a Taq DyeDeoxy Terminator Cycle Sequencing Kit (Applied Biosystems Inc., Foster City, Calif.) and an automated sequencer (Applied Biosystems Inc.; model 373A). The new sequences were compared with sequences of reference organisms, which were obtained from the EMBL and GenBank data libraries. Once the closest known phylogenetic relatives had been established, sequences were aligned by using the PILEUP program (3), and the alignments were corrected manually. For comparative phylogenetic analyses approximately 100 bases at the $5^{\prime}$ end of the rRNA sequence were excluded because of alignment uncertainties. Phylogenetic analyses were performed with the PHYLIP package (4). A distance matrix was calculated by using the program DNADIST, and a phylogenetic tree was calculated by the neighborjoining method with the program NEIGHBOR. The stability of the relationships was assessed by the bootstrap method with the programs SEQBOOT, DNADIST, NEIGHBOR, and CONSENSE.

The partial 16S rRNA gene sequence of $D$. pneumosintes ATCC $33048^{\mathrm{T}}$ consisted of 1,504 nucleotides (corresponding to positions 29 to 1504 of the Escherichia coli 16S rRNA). Sequence similarity calculations revealed that the closest known phylogenetic relatives of $D$. pneumosintes are the gramnegative organisms of the Sporomusa subbranch of the grampositive bacteria (approximately 81 to $88 \%$ sequence similarity). Members of the $B$. fragilis group of species were phylogenetically far removed from $D$. pneumosintes, exhibiting approximately 70 to $72 \%$ sequence similarity (data not shown). Table 1 shows the percent sequence similarities of $D$. pneumosintes with members of the Sporomusa subbranch. A distance matrix tree based on calculated evolutionary distances is shown in Fig. 1 and places $D$. pneumosintes within the Sporomusa subbranch of the Clostridium subphylum. It is evident from both distance calculations and the treeing program that within the Sporomusa subbranch, the closest known relatives of $D$. pneumosintes are Megasphaera elsdenii and the human oral Veillonella species. The significance of this grouping was measured by bootstrapping (Fig. 1). The sequence divergences observed between $D$. pneumosintes and $M$. elsdenii (approximately $11.5 \%$ ) and between $D$. pneumosintes and Veillonella species (12.2 to $12.8 \%)$ are clearly indicative of separate genera.

The results of the present study support the assignment of $B$. pneumosintes to a new genus, Dialister (11). Moore and Moore (11) placed Dialister in the family Bacteroidaceae, inasmuch as 
TABLE 1. Similarity values for the $16 \mathrm{~S}$ rRNA genes of $D$. pneumosintes and other members of the Sporomusa subbranch of the Clostridium subphylum of the gram-positive bacteria

\begin{tabular}{|c|c|c|c|c|c|c|c|c|c|c|c|c|c|c|}
\hline \multirow[b]{2}{*}{ Taxon } & \multicolumn{14}{|c|}{$\%$ Similarity with ${ }^{a}$ : } \\
\hline & 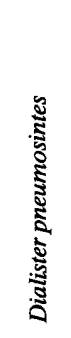 & 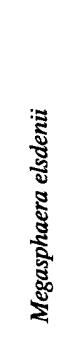 & 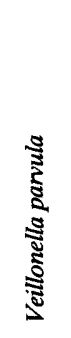 & 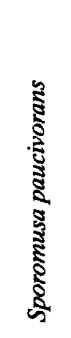 & 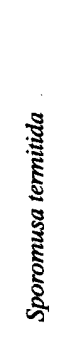 & 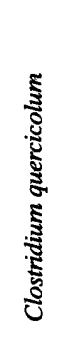 & 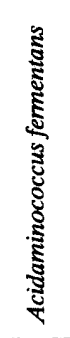 & 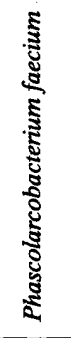 & 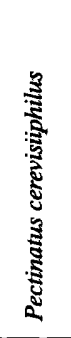 & 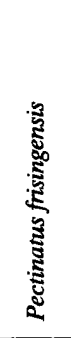 & 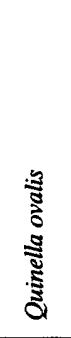 & 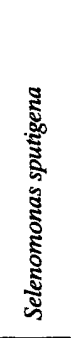 & 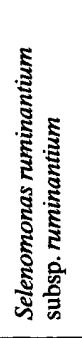 & 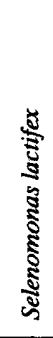 \\
\hline Megasphaera elsdenii & 88.5 & & & & & & & & & & & & & \\
\hline Veillonella parvula & 87.7 & 89.6 & & & & & & & & & & & & \\
\hline Sporomusa paucivorans & 84.3 & 84.6 & 85.9 & & & & & & & & & & & \\
\hline Sporomusa termitida & 81.4 & 83.4 & 84.2 & 93.2 & & & & & & & & & & \\
\hline Clostridium quercicolum & 83.8 & 86.4 & 86.9 & 90.0 & 88.0 & & & & & & & & & \\
\hline Acidaminococcus fermentans & 84.9 & 84.9 & 86.3 & 87.6 & 85.5 & 86.2 & & & & & & & & \\
\hline Phascolarcobacterium faecium & 83.9 & 85.7 & 86.1 & 89.0 & 86.9 & 88.2 & 90.6 & & & & & & & \\
\hline Pectinatus cerevisiiphilus & 85.2 & 86.4 & 87.0 & 88.1 & 86.0 & 88.1 & 85.4 & 86.2 & & & & & & \\
\hline Pectinatus frisingensis & 84.4 & 86.4 & 85.6 & 86.3 & 85.2 & 86.8 & 84.1 & 84.8 & 95.0 & & & & & \\
\hline Quinella ovalis & 81.8 & 81.9 & 81.9 & 82.8 & 81.4 & 84.0 & 82.8 & 83.7 & 83.9 & 83.4 & & & & \\
\hline Selenomonas sputigena & 84.8 & 85.4 & 85.6 & 86.2 & 84.6 & 88.3 & 86.8 & 86.5 & 88.7 & 88.9 & 85.5 & & & \\
\hline $\begin{array}{l}\text { Selenomonas ruminantium subsp. } \\
\text { ruminantium }\end{array}$ & 85.9 & 85.8 & 86.6 & 87.1 & 85.5 & 88.6 & 87.3 & 87.1 & 89.4 & 88.4 & 85.7 & 90.9 & & \\
\hline Selenomonas lactifex & 85.0 & 86.2 & 87.4 & 88.6 & 86.9 & 90.3 & 87.7 & 87.4 & 89.0 & 88.4 & 84.9 & 90.0 & 93.9 & \\
\hline Zymophilus paucivorans & 84.0 & 85.9 & 86.9 & 88.2 & 88.6 & 89.0 & 86.3 & 86.4 & 88.2 & 88.4 & 82.4 & 88.5 & 89.8 & 91.9 \\
\hline
\end{tabular}

${ }^{a} \mathrm{~A}$ continuous stretch of 1,259 nucleotides was used for the calculations.

it is "composed of anaerobic, gram-negative, nonsporing bacilli." However, this description also covers most of the taxa of the Sporomusa subbranch $(1,2)$. The family Bacteroidaceae is phylogenetically a member of the Flavobacterium-Cytophaga phylum of the gram-negative bacteria. It is clear, however, that several genera included in the Bacteroidaceae as currently defined (7) are phylogenetically far removed from the genus Bacteroides and in fact have a closer affinity to the Clostridium subphylum of the gram-positive bacteria (e.g., Fusobacterium and Leptotrichia [1], Butyrivibrio [5], and Pectinatus and Seleno- monas [6]). Therefore, a more specific definition of the family Bacteroidaceae, restricted to those genera (e.g., Porphyromonas and Prevotella) that are phylogenetically closely related to the genus Bacteroides, is needed. Although much work is still required before the higher taxonomic groupings within the Clostridium subphylum can be resolved with confidence, it is evident from the phylogenetic data presented here that the genus Dialister should be accommodated in the suprageneric grouping currently termed the Sporomusa subbranch.

Nucleotide sequence accession numbers. The 16S rRNA

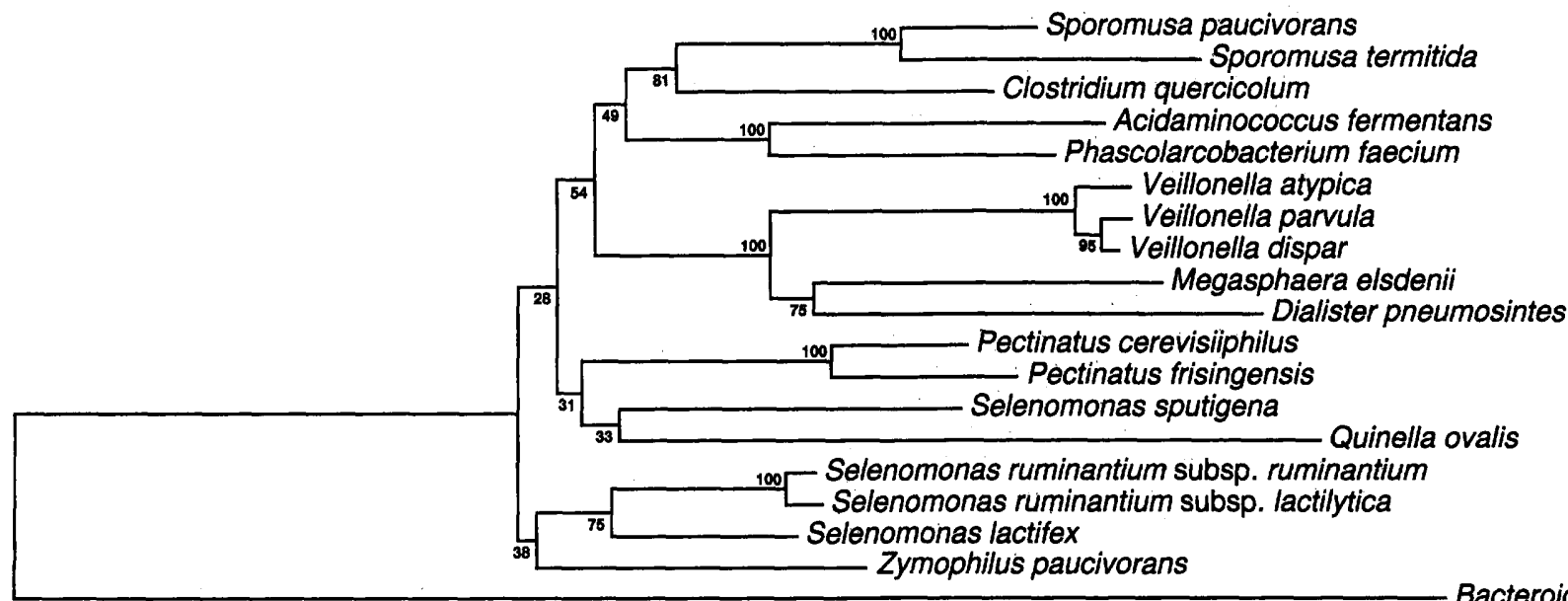

Bacteroides fragilis

FIG. 1. Unrooted phylogenetic tree showing the position of $D$. pneumosintes within the Sporomusa subbranch of the Clostridium subphylum of the gram-positive bacteria. Bootstrap values are given at the branching points. Values of 90 or above are considered significant. The bar represents a $2 \%$ sequence difference. 
gene sequences of $D$. pneumosintes ATCC $33048^{\mathrm{T}}, V$. atypica DSM $20739^{\mathrm{T}}, V$. dispar DSM $20735^{\mathrm{T}}$, and $V$. parvula DSM $2008^{\mathrm{T}}$ have been deposited in the EMBL data base under accession numbers X82500, X84007, X84006, and X84005, respectively.

\section{REFERENCES}

1. Collins, M. D., P. A. Lawson, A. Willems, J. J. Cordoba, J. FernandezGarayzabal, P. Garcia, J. Cai, H. Hippe, and J. A. E. Farrow. 1994. The phylogeny of the genus Clostridium: proposal of five new genera and eleven new species combinations. Int. J. Syst. Bacteriol. 44:812-826.

2. Del Dot, T., R. Osawa, and E. Stackebrandt. 1993. Phascolarcobacterium faecium gen. nov., a novel taxon of the Sporomusa group of bacteria. Syst. Appl. Microbiol. 16:380-384.

3. Devereux, J., P. Haeberli, and D. Smithies. 1984. A comprehensive set of sequence analysis programs for the VAX. Nucleic Acids Res. 12:387-395.

4. Felsenstein, J. 1989. PHYLIP-phylogeny inference package (version 3.2). Cladistics 5:164-166.

5. Hespell, R. B. 1992. The genera Butyrivibrio, Lachnospira, and Roseburia, p. 2022-2033. In A. Balows, H. G. Trüper, M. Dworkin, W. Harder, and K.-H. Schleifer (ed.), The prokaryotes. A handbook on the biology of bacteria: ecophysiology, isolation, identification, application. Springer-Verlag, New York.

6. Hespell, R. B., B. J. Paster, and F. E. Dewhirst. 1992. The genus Selenomonas, p. 2005-2013. In A. Balows, H. G. Trüper, M. Dworkin, W. Harder, and K.-H. Schleifer (ed.), The prokaryotes. A handbook on the biology of bacteria: ecophysiology, isolation, identification, application. Springer-Verlag, New York.
7. Holdeman, L. V., R. W. Kelley, and W. E. C. Moore. 1984. Genus 1. Bacteroides Castellani and Chalmers 1919, p. 604-631. In N. R. Krieg and J. G. Holt (ed.), Bergey's manual of systematic bacteriology, vol. 1. The Williams and Wilkins Co., Baltimore.

8. Holdeman, L. V., and W. E. C. Moore. 1970. Outline of clinical methods in anaerobic microbiology, 2nd rev., p. 33. Virginia Polytechnic Institute and State University Anaerobe Laboratory, Blacksburg.

9. Hutson, R. A., D. E. Thompson, and M. D. Collins. 1993. Genetic interrelationships of saccharolytic Clostridium botulinum types B, E and F and related clostridia as revealed by small subunit rRNA sequences. FEMS Microbiol. Lett. 108:103-110.

10. Lawson, P. A., S. E. Gharbia, H. N. Shah, and D. R. Clark. 1989. Recognition of Fusobacterium nucleatum subgroups $\mathrm{Fn}-1, \mathrm{Fn}-2$ and $\mathrm{Fn}-3$ by ribosomal RNA gene restriction patterns. FEMS Microbiol. Lett. 65:41-46.

11. Moore, L. V. H., and W. E. C. Moore. 1994. Oribaculum catoniae gen. nov., sp. nov.; Catonella morbi gen. nov., sp. nov.; Hallella seregens gen. nov., sp. nov.; Johnsonella ignava gen. nov., sp. nov.; and Dialister pneumosintes comb. nov., nom. rev., anaerobic gram-negative bacilli from the human gingival crevice. Int. J. Syst. Bacteriol. 44:187-192.

12. Olitsky, P. K., and F. L. Gates. 1921. Experimental studies of the nasopharyngeal secretions from influenza patients. J. Exp. Med. 33:713-729.

13. Shah, H. N. 1992. The genus Bacteroides and related taxa, p. 3593-3607. In A. Balows, H. G. Trüper, M. Dworkin, W. Harder, and K.-H. Schleifer (ed.), The prokaryotes. A handbook on the biology of bacteria: ecophysiology, isolation, identification, application. Springer-Verlag, New York.

14. Shah, H. N., and M. D. Collins. 1989. Proposal to restrict the genus Bacteroides (Castellani and Chalmers) to Bacteroides fragilis and closely related species. Int. J. Syst. Bacteriol. 39:85-87. 